\title{
Teste Propósito de Vida: Propriedades Psicométricas e Evidências de Validade
}

\author{
Raul Bruno Tibaldi Nascimento, Tatiane Lebre Dias ${ }^{1}$ \\ Universidade Federal de Mato Grosso, Cuiabá-MT, Brasil
}

\section{RESUMO}

O sentido de vida é o pressuposto fundamental da Logoterapia e Análise Existencial, e o PIL-Test (Teste Propósito de Vida) tem se consolidado como um dos instrumentos mais relevantes para sua mensuração. Este estudo analisou a estrutura fatorial desse instrumento, cujas análises ainda sustentam uma controvérsia quanto ao modelo mais consistente para sua explicação. Participaram da pesquisa 200 acadêmicos de Psicologia, com média de 23,62 anos $(D P=7,071)$. Além do poder discriminativo, homogeneidade e consistência interna dos itens do instrumento, realizou-se uma análise fatorial exploratória a partir de matriz de correlações policóricas, com o estimador DWLS. O estudo reuniu evidências psicométricas satisfatórias e de uma estrutura de um fator para a matriz dos itens, com índices de ajuste adequados, além de corroborar sugestões prévias quanto à exclusão de certos itens para uma estrutura mais reduzida e parcimoniosa.

Palavras-chave: sentido de vida; logoterapia; análise fatorial.

\section{ABSTRACT - Purpose in Life Test: Psychometric Properties and Validity Evidence}

The meaning of life is the central assumption of Logotherapy and Existential Analysis and the PIL-Test (Purpose in Life Test) has been consolidated as one of the most relevant instruments for its assessment. This study examined the instrument's factorial structure, whose analyses still sustain a controversy on the most reliable fit to explain it. A total of 200 Psychology students participated in the study, with average age of 23.62 years $(S D=7.071)$. Besides investigating the discriminant power, homogeneity and internal consistency of the instrument's items, an exploratory factorial analysis was conducted based on the polychoric correlation matrix and the DWLS estimation. The study gathered satisfactory psychometric evidence, indicating a one-factor structure for the matrix of the items with acceptable fit indices, as well as corroborating previous suggestions regarding the exclusion of certain items aimed at a smaller and more parsimonious structure.

Keywords: meaning of life; logotherapy; factor analysis.

\section{RESUMEN - Purpose in Life Test: Propiedades Psicométricas y Evidencias de Validez}

El sentido de vida es el supuesto fundamental de la Logoterapia y el Análisis Existencial y el PIL-Test (Test de Propósito Vital) viene siendo consolidado como uno de los instrumentos más relevantes para su medición. Este estudio averiguó la estructura factorial de ese instrumento, cuyas análisis aún sostienen una controversia en cuanto al modelo más consistente para su explicación. Participaron de la investigación 200 académicos de Psicología, con promedio de 23,62 años ( $D S=7,071)$. Además del poder discriminatorio, homogeneidad y consistencia interna de los ítems del instrumento, se realizó un análisis factorial exploratorio a partir de matriz de correlaciones policóricas, con el estimador DWLS. El estudio reunió evidencias psicométricas satisfactorias y de una estructura de un factor para la matriz de los ítems, con índices de ajuste adecuados, además de corroborar sugerencias previas en cuanto a la exclusión de ciertos ítems para una estructura más reducida y parsimoniosa.

Palabras clave: sentido de la vida; logoterapia; análisis factorial.

O questionamento sobre o sentido da vida pode ser abordado como um fenômeno tipicamente humano, na medida em que se trata de uma indagação que muitos indivíduos sentem a necessidade de responder. Em Psicologia, a Logoterapia é a teoria cuja ênfase central está fundamentada na orientação do homem para realização de um sentido na vida (Xausa, 2011). Conhecida como a Terceira Escola Vienense de
Psicoterapia e desenvolvida pelo psiquiatra austríaco Viktor E. Frankl, a Logoterapia pode ser compreendida como uma psicoterapia centrada no sentido. Com efeito, o termo de origem grega logos significa "sentido", referindo-se, nesse caso, ao sentido da existência humana. Segundo Frankl, o ser humano é, primariamente, motivado pela busca por esse sentido na vida (Frankl, 1985/2016). 
Entre as bases da Logoterapia, encontra-se a concepção de que há uma "vontade de sentido" (Frankl, 1985/2016, p. 124) que perpassa o ser humano e que neste será frustrada sempre que for acometido de falta de sentido e de vazio existencial. A vontade de sentido decorre da característica antropológica da autotranscendência, pela qual a existência humana aponta para algo ou para alguém, ou seja, para um sentido que deve ser preenchido ou para a existência de outro ser humano que encontra (Frankl, 1956/2016).

A partir da década de 1960, contribuiu para a consolidação dessa teoria a condução de pesquisas que se propuseram, dentre outros objetivos, à construção e validação de instrumentos psicométricos de mensuração do sentido de vida, ressaltando-se o pioneirismo do Teste Propósito de Vida (Purpose in Life Test - PIL) e o Teste de Busca de Objetivos Noogênicos (Seeking of Noetic Goals Test-SONG) (Pacciolla, 2016). Além desses, podem ser citados o Logo-Teste (Lukas, 1988, citado por Aquino et al., 2015), o Meaning in Life Questionnaire (Steger, Frazier, Oishi, \& Kaler, 2006), o Life Engagement Test (Scheier et al., 2006), o Schedule for Meaning in Life Evaluation SMiLE (Fegg, Kramer, L'hoste, \& Borasio, 2008), o Sources of Meaning and Meaning in Life Questionnaire SoMe (Schnell, 2009) e a Escala Dimensional del Sentido de Vida (Martinez Ortiz, Trujillo, \& Diaz Del Castillo, 2011), entre outros.

Nesse contexto, destaca-se a relevância do Teste Propósito de Vida (Purpose in Life Test - PIL Test), que procura medir o nível de sentido de vida alcançado por um indivíduo, especialmente por se tratar de um dos principais testes cujos padrões psicométricos são objeto de extensa análise por membros da comunidade científica e acadêmica voltada ao desenvolvimento da Logoterapia.

Esse instrumento foi originalmente desenvolvido por James C. Crumbaugh e Leonard T. Maholick, na década de 60 , considerando que não havia tentativas consistentes de mensuração dos fenômenos chamados por Frankl de "neuroses noogênicas", embora ele os houvesse delineado como resultantes da completa falta de sentido de vida nos indivíduos. Crumbaugh e Maholick (1964) apresentaram um estudo com o objetivo de proceder à quantificação do conceito de "propósito" ou "sentido na vida" (p. 201), por meio de um instrumento que lhe fosse adequadamente sensível. Nesse sentido, os autores optaram pela definição de "propósito na vida" como "o significado ontológico da vida do ponto de vista da experiência individual” (p. 201).

Em termos de estrutura, o teste foi constituído por uma escala de atitudes composta por 22 itens, respondidos por meio de uma escala do tipo Likert de sete pontos, a fim de evocar respostas presumivelmente relacionadas ao grau de propósito na vida experienciado pelos participantes. A pontuação total era obtida pela soma das pontuações em cada item. Após a análise das respostas de uma amostra de 225 pessoas, entre elas estudantes e pacientes hospitalizados, os resultados indicaram que o PIL-Test era capaz de avaliar os aspectos do que Frankl chamou de "frustração existencial", justamente pela falta de sentido na vida, além de fornecerem evidências da validade dos construtos básicos da Logoterapia (Crumbaugh \& Maholick, 1964).

Dando continuidade às investigações psicométricas relativas aos conceitos básicos da teoria, Reker e Cousins (1979) buscaram, entre outros objetivos, examinar a estrutura fatorial dos itens do PIL-Test e avaliar sua consistência interna e homogeneidade. Nesse trabalho, os autores utilizaram a versão mais conhecida e atual do instrumento, consolidada por Crumbaugh e Maholick (1968, 1969), que é composta por 20 itens avaliados numa escala de 1 a 7 pontos. Reker e Cousins (1979) explicam que, quanto maiores as pontuações, maiores são os indícios de um alto nível de sentido na vida, da mesma forma que pontuações baixas são indicativas de um baixo nível de sentido na vida. As pontuações foram submetidas a uma análise dos componentes principais com rotação varimax, estabelecendo-se como critérios valores próprios (eigenvalues) maiores que 1 e correlações dos itens superiores a 0,30 . Diante dos resultados, reuniram-se fortes evidências da validade fatorial do teste, composta por seis fatores, denominados pelos autores como "Propósito na Vida", "Satisfação com a Vida", "Concretização de Objetivos", "Autossuficiência", "Lócus de Controle Interno-Externo" e "Perspectiva de Vida".

Interessados em avaliar as propriedades psicométricas do PIL-Test, Harlow, Newcomb e Bentler (1987) também exploraram a estrutura fatorial dos itens desse teste numa ampla amostra de população de jovens adultos, por meio do método da análise fatorial por Máxima Verossimilhança com rotação oblíqua. Ao final, concluiu-se que o instrumento aparentava possuir quatro fatores primários - "Propósito na vida", "Ausência de sentido", "Felicidade" e "Predisposição ao suicídio" -, bem como um amplo fator geral. Resultados semelhantes foram ratificados num estudo com 194 mulheres conduzido por Chamberlain e Zika (1988), que examinaram três escalas de mensuração do sentido na vida, incluindo o PILTest. Para essa escala, uma Análise dos Componentes Principais levou à extração de quatro fatores relacionados aos aspectos de (1) sentido, (2) satisfação, (3) controle e (4) entusiasmo na vida. Entretanto, uma posterior análise dos eixos principais possibilitou identificar um fator geral, denominado "sentido na vida", para o qual não contribuíram com carga fatorial mínima apenas os itens 7, 13 e 15 do teste. Da mesma forma, as análises realizadas por Noblejas de la Flor (1999) e por Su, Chen, Chen, Yang e Hung (2006) proporcionaram quatro fatores na estrutura interna do PIL-Test.

Em contrapartida, Valladares, Zavala, Tarango, Medina e Medina (2004), ao explorarem as relações entre sentido na vida e outras variáveis relativas a estudantes universitários mexicanos, observaram uma 
escala constituída por três fatores: "Percepção de sentido e significado da vida", "Satisfação pela própria Vida" e "Liberdade e Controle da Vida". Nesse caso, procedeu-se a uma análise fatorial exploratória utilizando o método dos componentes principais com rotação varimax. Em outro contexto, Jonsén et al. (2010) também verificaram uma estrutura similar do PIL-Test, tendo nomeado os fatores por "Sentido da existência", "Liberdade para criar sentido na vida diária" e "Vontade de encontrar sentido em desafios futuros". Nesse estudo, os itens 12, 13 e 16 não apresentaram carga fatorial adequada em nenhum dos três fatores. No Brasil, um estudo correlacional entre atitude religiosa e sentido da vida delineado por Aquino et al. (2009) sugeriu a presença de três fatores, obtidos por uma análise fatorial dos eixos principais com rotação varimax, intitulados "vazio existencial", "realização existencial" e "desespero existencial".

Ademais, soluções de dois fatores foram obtidas em diversas pesquisas a respeito do PIL-Test. Dufton e Perlman (1986), por exemplo, abordaram a homogeneidade desse instrumento, testando a hipótese de associação entre religiosidade e propósito na vida numa amostra composta por universitários canadenses de Psicologia. Após conduzirem uma Análise dos Componentes Principais com rotação varimax, os autores consideraram mais apropriada a extração de dois fatores, "Satisfação com a vida" e "Propósito na vida", sendo o primeiro o responsável pela maior variação nas pontuações totais. Os itens 7, 13, 14, 15, 16 e 18, contudo, não apresentaram carga fatorial suficiente para nenhum deles. Do mesmo modo, dois fatores dominantes foram identificados nos trabalhos de Morgan e Farsides (2009) e de GarcíaAlandete, Martínez e Nohales (2013). Destaca-se que os modelos de dois fatores do PIL-Test foram, inclusive, descritos como mais ajustados e confiáveis, após análises fatoriais confirmatórias procedidas por Martínez, García-Alandete, Nohales, Valero e Lozano (2012), cujos resultados possibilitaram sugerir uma versão reduzida do instrumento que inclua um fator geral do sentido e satisfação na vida e outro que reuniria os respectivos itens relacionados com propósitos e metas individuais. No Brasil, contribuíram no mesmo sentido as pesquisas de Melo, Eulálio, Silva, Silva Filho e Gonzaga (2013) e de Nobre (2016), que encontraram dois fatores para explicar a estrutura e consistência interna do PIL-Test, com índices estatísticos semelhantes.

Os achados de Marsh, Smith, Piek e Saunders (2003), por sua vez, corroboraram estudos que advogaram pela unidimensionalidade do PIL-Test. Num exame das suas propriedades psicométricas a partir das respostas de consumidores de bebidas alcoólicas, o modelo de fator único se mostrou adequado pela análise fatorial confirmatória, com a exclusão dos itens 7, 14 e 15. No Brasil, Aquino (2009) adotou uma solução de um fator para o PIL-Test, priorizando a parcimônia e baseando-se em estudos prévios, apesar de admitir ser possível a multidimensionalidade do teste. Nesse estudo, um grande fator geral, denominado "vazio existencial", apresentou resultados consistentes, levando, porém, à sugestão de exclusão dos itens $1,7,11$, 12, 13, 14, 15, 17 e 18. De fato, García-Alandete, Marco e Pérez (2017) concluíram, mais recentemente, que a escala composta por apenas um fator se mostrou a mais parcimoniosa em comparação aos outros principais modelos propostos para o instrumento até então.

De modo geral, apesar de os estudos terem obtido índices consistentes em relação às propriedades psicométricas do PIL-Test, ratificando a sensibilidade do instrumento ao que se propõe mensurar, percebe-se uma forte controvérsia no tocante à estrutura interna do instrumento. Ainda que achados mais recentes tenham proposto a adequabilidade e melhor ajuste dos modelos de um e dois fatores para explicá-la plausivelmente, não foi possível estabelecer um consenso em favor de um deles, o que, talvez, possa ser explicado pela não utilização de uma combinação de estimadores que conferissem maior robustez às análises estatísticas na determinação do número de dimensões do instrumento.

Diante disso, e considerando a relativa escassez de trabalhos que priorizem a validação dos padrões psicométricos desse teste no contexto brasileiro, não obstante sua reconhecida importância para a comunidade científica e acadêmica, este estudo buscou analisar a estrutura fatorial do PIL-Test, além de averiguar suas propriedades psicométricas de validade e precisão.

\section{Método}

\section{Participantes}

Participaram da pesquisa 200 estudantes de um curso de Psicologia da cidade de Cuiabá/MT, com idade média de 23,62 anos $(D P=7,071)$, dos quais $76 \%$ eram do sexo feminino. Dos participantes, $41 \%$ se declararam brancos, $38,5 \%$, pardos, e $17,5 \%$, negros. $66,5 \%$ deles declararam não exercer nenhuma atividade remunerada e apenas 9,5\% declararam morar sozinhos.

\section{Instrumentos}

Os participantes preencheram um questionário de perfil socioeconômico, abrangendo aspectos relativos a idade, cor/etnia, vínculo empregatício e moradia, entre outros. Além disso, responderam ao Teste Propósito de Vida (PIL-Test) na versão brasileira atual, que é composta por 20 itens, respondidos por meio de escala Likert de 1 a 7 pontos, num intervalo de "discordo totalmente" a "concordo totalmente", respectivamente, conforme consta em trabalhos nacionais (Aquino, 2009; Aguiar, 2011; Nobre, 2016). Os itens são discriminados na seção dos Resultados.

\section{Procedimentos}

A pesquisa foi autorizada pela respectiva coordenação de curso e aprovada no Comitê de Ética em Pesquisa 
da Universidade de Cuiabá (número do parecer: 1.762.932). A coleta dos dados foi realizada nas salas de aula dos dez semestres constituintes do curso, durante o período de dois dias. A participação foi voluntária e confirmada após a leitura e assinatura, pelos estudantes, de um Termo de Consentimento Livre e Esclarecido acerca dos objetivos da pesquisa.

\section{Análise dos dados}

Os dados obtidos foram analisados por meio dos softwares estatísticos SPSS (versão 22) e Factor Analysis (versão 10.8.04). Para analisar o poder discriminativo dos itens do PIL-Test, optou-se pelo método de criação de dois grupos-critério. O primeiro foi constituído pelos sujeitos cujos escores totais estavam acima da mediana; o segundo, por aqueles cujos escores totais estavam abaixo da mediana. Em seguida, empregou-se um teste $\mathrm{t}$ para avaliar a diferenciação de cada item. A homogeneidade dos itens foi analisada por meio da verificação das correlações dos itens com os escores totais do teste. A confiabilidade da consistência interna do teste foi constatada por coeficiente alfa de Cronbach de 0,84 (Cohen, Swerdlik, \& Sturman, 2014; Nobre, 2016).

$\mathrm{A}$ amostra se mostrou adequada à fatorabilidade da matriz dos itens diante dos índices $\mathrm{KMO}=0,85$ e Teste de Esfericidade de Bartlett, $\chi^{2}(190)=1249,9 ;(p<0,001)$. Confirmada a possibilidade da análise fatorial exploratória, o número de fatores da matriz de dados foi determinado a partir da utilização do método de Análises
Paralelas com permutação de valores (Timmerman \& Lorenzo-Seva, 2011) e do método Hull (LorenzoSeva, Timmerman, \& Kiers, 2011). Realizou-se, então, uma análise fatorial por meio da matriz de correlações policóricas, em combinação com o estimador DWLS (Diagonally Weighted Least Squares) (Asún, Rdz-Navarro, \& Alvarado, 2015), estabelecendo como critério cargas fatoriais iguais ou superiores a 0,40 .

Por fim, para se comprovar o ajuste do modelo da estrutura interna do instrumento, foram utilizados os seguintes índices: o Root Mean Square Error of Approximation (RMSEA), considerando valor igual ou menor a 0,06 como indicativo de ajuste adequado; o $\chi^{2}(g l)$; o Non-Normed Fit Index (NNFI; Tucker \& Lewis), considerando valor igual ou maior a 0,95 como indicativo de ajuste adequado; o Comparative Fit Index (CFI) e o Goodness of Fit Index (GFI), considerando, para ambos, valor igual ou maior a 0,90 como indicativos de adequação ao modelo, ao nível de significância de 0,05 .

\section{Resultados}

Os resultados indicaram que os itens 7, 11, 15 e 17 do Teste Propósito de Vida não apresentaram poder discriminativo satisfatório $(p<0,001)$ no teste $\mathrm{t}$ entre as médias dos grupos-critério internos. Além disso, esses mesmos itens apresentaram desempenho abaixo do limite aceitável (ri.t.<0,20) quanto às correlações item-total corrigidas.

Tabela 1

Análises Paralelas baseada em Minimum Rank Factor Analysis

\begin{tabular}{cccc}
\hline Fatores & \% variância empírica & $\begin{array}{c}\text { \% variância da } \\
\text { média aleatória }\end{array}$ & $\begin{array}{c}\text { \% variância do } 95^{\circ} \\
\text { percentil aleatório }\end{array}$ \\
\hline 1 & $42,65^{*}$ & 12,86 & 14,40 \\
2 & 10,81 & 11,73 & 12,94 \\
3 & 7,85 & 10,77 & 11,75 \\
4 & 6,60 & 9,90 & 10,77 \\
5 & 5,62 & 9,02 & 9,80 \\
\hline
\end{tabular}

Nota. Número de 500 matrizes aleatórias de correlação policórica. Método permutação de dados brutos (Buja \& Eyuboglu, 1992). *Número de fatores recomendado. Apenas os cinco primeiros fatores são mostrados

Ambos os métodos empregados para extração do número de fatores indicaram a presença de um fator, conforme descrito nas tabelas 1 e 2 , segundo o critério de análises paralelas e o método Hull, respectivamente.

Tabela 2

Índices de Ajuste Apresentados por Diferentes Soluções Fatoriais segundo o Critério HULL-CFI

\begin{tabular}{cccc}
\hline$N^{\circ}$ fatores & CFI & gl & Valores no Scree-test \\
\hline 0 & 0,00 & 190 & 0,00 \\
1 & 0,96 & 170 & $56,60^{*}$ \\
2 & 0,98 & 151 & 1,84 \\
3 & 0,99 & 133 & 0,00 \\
\hline
\end{tabular}

Nota. *Número de fatores recomendado 
Desse modo, adotando-se a solução de um fator, realizou-se a análise fatorial exploratória, na qual se observou que a maioria dos itens apresentou carga fatorial satisfatória, com exceção dos itens 7,11 , 13, 14, 15, 17 e 18, conforme apresentado na Tabela 3. O fator extraído apresentou valor próprio de 6,82 , explicando34,11\%davariânciatotal, comalfade Cronbach total de 0,88 .

Observou-se que o modelo de um fator apresentou boa adequação, conforme os índices considerados [RMSEA $=0,06($ IC $95 \%=0,050-0,080) ; \mathrm{NNFI}=0,96$; $\left.\mathrm{CFI}=0,96 ; \mathrm{GFI}=0,97 ; \chi^{2}(170)=251,85\right]$.

Tabela 3

Estrutura Fatorial do Teste Propósito de Vida (PIL-Test)

\begin{tabular}{lcc}
\hline \multicolumn{1}{c}{ Item } & Saturação & $h^{2}$ \\
\hline 1. Geralmente, estou completamente aborrecido. & $-0,59^{*}$ & 0,35 \\
2. A vida para mim parece sempre empolgante. & $-0,61^{*}$ & 0,38 \\
3. Tenho na vida metas e objetivos muito claros. & $-0,51^{*}$ & 0,26 \\
4. Minha experiência pessoal é inteiramente sem sentido ou propósito. & $-0,73^{*}$ & 0,54 \\
5. Todo dia é constantemente novo. & $-0,57^{*}$ & 0,32 \\
6. Se eu pudesse escolher, preferiria nunca ter nascido. & $-0,79^{*}$ & 0,62 \\
7. Após a aposentadoria, faria algumas das coisas empolgantes que sempre quis fazer. & $-0,19$ & 0,03 \\
8. Quanto a alcançar metas na vida, não tenho feito nenhum progresso. & $-0,58^{*}$ & 0,34 \\
9. Minha vida é vazia, preenchida só com desespero. & $-0,83^{*}$ & 0,68 \\
10. Se eu morresse hoje, sentiria que minha vida foi muito valiosa. & $-0,68^{*}$ & 0,46 \\
11. Ao pensar em minha vida, frequentemente penso por que eu existo. & $-0,27$ & 0,07 \\
12. Considerando o mundo em relação a minha vida, o mundo deixa-me totalmente confuso. & $-0,64^{*}$ & 0,41 \\
13. Eu não sou uma pessoa muito responsável. & $-0,28$ & 0,08 \\
14. Quanto à liberdade do homem para tomar suas próprias decisões, acredito que o homem & $-0,28$ & 0,08 \\
é totalmente livre para fazer todas as escolhas da vida. & & 0,01 \\
15. Quanto à morte, estou preparado e sem medo. & 0,00 \\
16. Quanto ao suicídio, tenho pensado seriamente ao seu respeito como uma saída. & $-0,73^{*}$ & 0,54 \\
17. Considero a possibilidade de encontrar um sentido, um propósito ou missão em minha & $-0,17$ & 0,03 \\
vida como muito grande. & $-0,34$ & 0,12 \\
18. Minha vida está em minhas mãos e eu a controlo. & $-0,73^{*}$ & 0,53 \\
19. Encarar minhas tarefas diárias é uma fonte de prazer e satisfação. & $-0,69^{*}$ & 0,47 \\
20. Não descobri qualquer missão ou propósito na vida. & \\
\hline
\end{tabular}

Nota. * carga fatorial considerada satisfatória (maior ou igual a 0,40)

\section{Discussão}

Por um lado, estima-se que o presente estudo tenha obtido uma explicação mais consistente da estrutura fatorial do instrumento analisado, por meio de uma combinação de métodos que tendem a ser mais robustos a violações de normalidade. Entre os estudos analisados, apenas Marsh, Smith, Piek e Saunders (2003) utilizaram uma combinação similar para a análise da estrutura fatorial do PIL-Test, obtendo, também, um modelo unidimensional satisfatório. Outrossim, corrobora-se a sugestão de uma estrutura reduzida e mais parcimoniosa para o instrumento, em que pesem divergências quanto aos itens a serem excluídos do teste.

Nesse sentido, a exclusão dos itens 7 (aposentadoria), 15 (medo da morte) e 17 (expectativa de realização de sentido) poderia ser justificada, à luz da teoria de base do instrumento, por se tratar de itens que provavelmente não remetem a situações concretas aos indivíduos, estando associados mais fortemente a perspectivas futuras. Segundo Frankl (1969/2011, p. 81), "sentido é o que se tenciona, seja por uma pessoa que me pergunta algo, seja por uma situação que encerra uma pergunta e clama por uma resposta". Trata-se de um sentido concreto 
percebido numa situação "com a qual uma pessoa igualmente concreta é respectivamente confrontada" (Frankl, 2012, p. 283). Xausa (2011) reforça esse aspecto do conceito de sentido, referindo-se a ele como uma potencialidade latente que deve ser encontrada em cada situação. A exclusão dos itens 11, 13, 14 e 18, por sua vez, poderia ser explicada por estarem relacionados a ideias que talvez não sejam compreendidas, pelos indivíduos, como intrínsecos à realização de sentido de vida. Sabe-se que a compreensão, em Logoterapia e Análise Existencial, de pressupostos como liberdade e responsabilidade, por exemplo, diverge, em alguma medida, da compreensão perpassada pelo senso comum e por aspectos semânticos dessas palavras.

Por outro lado, é possível citar como limitação deste estudo a caracterização da respectiva amostra, considerada de conveniência e composta essencialmente por adultos jovens que ainda não trabalham, inseridos num contexto restrito e bastante particular de uma universidade. Isso sugere a realização de outras pesquisas com amostras que representem mais amplamente a população geral.

Em resumo, este estudo reuniu evidências psicométricas favoráveis para se considerar o PIL-Test um instrumento sensível ao que busca investigar. Ao mesmo tempo, ressalta-se o fato de que instrumentos psicológicos não devem ser utilizados com fim em si mesmos, mas sim considerados dentro de um processo mais amplo de avaliação psicológica a fim de apreender qualquer fenômeno ou condição dos respectivos sujeitos. Nesse sentido, o PIL-Test se mostra pertinente como subsídio, por exemplo, para se levantar uma noção geral acerca do nível de realização de sentido de vida de indivíduos ou grupos, no intuito de planejar estratégias de intervenção envolvendo a temática.

\section{Referências}

Aguiar, A. A. (2001). Relações entre valores, sentido de vida e bem-estar subjetivo em membros de novas comunidades católicas (Dissertação de mestrado). Departamento de Ciências das Religiões, Universidade Federal da Paraíba, João Pessoa.

Aquino, T. A. A. (2009). Atitudes e intenções de cometer o suicídio: Seus correlatos existenciais e normativos (Tese de doutorado). Departamento de Psicologia, Universidade Federal da Paraíba, João Pessoa.

Aquino, T. A. A., Correia, A. P. M., Marques, A. L. C., Souza, C. G., Freitas, H. C. A., Araújo, I. F., Dias, P. S., \& Araújo, W. F. (2009). Atitude religiosa e sentido da vida: Um estudo correlacional. Psicologia Ciência e Profissão, 29(2), 228-243. doi: 10.1590/S141498932009000200003

Aquino, T. A. A., Veloso, V. G., Aguiar, A. A., Pereira, G. A., Fernandes, A. S., Serafim, T. D. B., \& Pontes, A. M. (2015). Questionário de Sentido de Vida: Evidências de sua validade fatorial e consistência interna. Psicologia: Ciência e Profissão, 35(1), 4-19. doi: 10.1590/19823703001332012

Asún, R. A., Rdz-Navarro, K., \& Alvarado, J. M. (2015). Developing Multidimensional Likert Scales using Item Factor Analysis: The Case of Four-Point Items. Sociological Methods \& Research, 45(1). doi: 10.1177/0049124114566716

Buja, A. \& Eyuboglu, N. (1992). Remarks on Parallel Analysis. Multivariate Behavioral Research, 27(4), 509-540. doi: 10.1207/ s15327906mbr2704_2

Chamberlain, K., \& Zika, S. (1988). Measuring meaning in life: An examination of three scales. Personality and Individual Differences, 9(3), 589-596. doi: 10.1016/0191-8869(88)90157-2

Cohen, R. J., Swerdlik, M. E., \& Sturman, E. D. (2014). Testagem e avaliação psicológica: Introdução a testes e medidas (8a ed., Monteiro, M. C G., Trad.). Porto Alegre: AMGH.

Crumbaugh, J. C., \& Maholick, L. T. (1964). An experimental study in existentialism: The psychometric approach to Frankl's concept of noogenic neurosis. Journal of Clinical Psychology, 20(2), 200-207. doi: 10.1002/1097-4679(196404)20:2<200::AIDJCLP2270200203>3.0.CO;2-U

Dufton, B. D., \& Perlman, D. (1968). The Association between Religiosity and the Purpose in-Life-Test: Does it Reflect Purpose or Satisfaction? Journal of Psychology and Theology, 14(1), 42-48. Recuperado de https://www.researchgate.net/publication/232604994_The_ association_between_religiosity_and_the_Purpose-in-Life_test_Does_it_reflect_purpose_or_satisfaction

Fegg, M. J., Kramer, M., L'hoste, S., \& Borasio, G. D. (2008, April). The Schedule for Meaning in Life Evaluation (SMiLE): Validation of a New Instrument for Meaning-in-Life Research. Journal of Pain and Symptom Management, 35(4), 356-364. doi: 10.1016/j. jpainsymman.2007.05.007

Frankl, V. E. (2011). A vontade de sentido: Fundamentos e aplicações da logoterapia (Pereira, I. S., Trad.). São Paulo: Paulus. (Original publicado em 1969).

Frankl, V. E. (2012). Logoterapia e análise existencial: Textos de seis décadas. (M. A. Casanova, Trad.). Rio de Janeiro: Forense Universitária.

Frankl, V. E. (2016). Em busca de sentido: Um psicólogo no campo de concentração (W. O. Schlupp \& C. C. Aveline, Trad.). 39a ed. São Leopoldo: Sinodal; Petrópolis: Vozes. (Original publicado em 1985).

Frankl, V. E. (2016). Teoria e terapia das neuroses: Introdução à logoterapia e à análise existencial (C. Abeling, Trad.). São Paulo: É Realizações. (Original publicado em 1956).

García-Alandete, J., Martínez, E. R., \& Nohales, P. S. (2013). Estructura factorial y consistencia interna de una versión española del PurposeIn-Life Test. Universitas Psychologica, 12(2), 517-530. doi: 10.11144/Javeriana.UPSY12-2.efci

García-Alandete, J., Marco, J. H., \& Pérez, S. (2017). Purpose-in-Life Test: Comparison of the Main Models in Patients with Mental Disorders. The Spanish Journal of Psychology, 20(31), 1-9. doi: 10.1017/sjp.2017.28

Harlow, L. L., Newcomb, M. D., \& Bentler, P. M. (1987). Purpose in Life Test assessment using latent variable methods. British Journal of Clinical Psychology, 26(3), 235-236. doi: 10.1111/j.2044-8260.1987.tb01355.x 
Jonsén, E., Fagerström, L., Lundman, B., Nygren, B.,Vähäkangas, M., \& Strandberg, G. (2010). Psychometric properties of the Swedish version of the Purpose in Life scale. Scandinavian Journal of Caring Sciences, 24(1), 41-48. doi: 10.1111/j.1471-6712.2008.00682.x

Lorenzo-Seva, U., Timmerman, M. E., \& Kiers, H. A. (2011). The hull method for selecting the number of common factors. Multivariate Behavioral Research, 46(2), 340-364. doi: 10.1080/00273171.2011.564527

Marsh, A., Smith, L., Piek, J., \& Saunders, B. (2003). The purpose in life scale: Psychometric properties for social drinkers and drinkers in alcohol treatment. Educational and Psychological Measurement, 63(5), 859-871. doi: 10.1177/0013164402251040

Martínez, E. R., García-Alandete, J., Nohales, P. S., Valero, G. B., \& Lozano, B. S. (2012). Análisis factorial confirmatorio de los principales modelos propuestos para el Purpose-in-LifeTest en una muestra de universitarios españoles. Acta Colombiana de Psicología, 15(1), 67-76. Recuperado de https://dialnet.unirioja.es/descarga/articulo/5801731.pdf

Martinez Ortiz, E., Trujillo, A. M., \& Diaz Del Castillo, J. P. (2011). Desarrollo y estructura de la Escala Dimensional del Sentido de Vida. Acta Colombiana de Psicología, 14(2), 113-119. Recuperado de http:/www.scielo.org.co/pdf/acp/v14n2/v14n2a11.pdf

Melo, R. L. P., Eulálio, M. C., Silva, H. D. M., Silva Filho, J. M., \& Gonzaga, P. S. (2013). Sentido de vida, dependência funcional e qualidade de vida em idosos. Revista Brasileira de Geriatria e Gerontologia, 16(2), 239-250. doi: 10.1590/S1809-98232013000200004

Morgan, J., \& Farsides, T. (2009). Measuring Meaning in Life. Journal of Happiness Studies, 10(2), 197-214. doi: 10.1007/s10902-007-9075-0

Noblejas de la Flor, M. A. (1999). Estructura factorial del test PIL y Logo-test. NOUS: Boletín de Logoterapia y Análisis Existencial, 3 , 67-84. Recuperado de http://manoblejas.eresmas.net/nous.htm\#NOUS3

Nobre, M.A. R. (2016). Purpose in Life Test (PIL-Test): Evidências de validade e precisão. Revista Logos E Existência: Revista da Associação Brasileira de Logoterapia e Análise Existencial, 5(1), 89-118. Recuperado de http://periodicos.ufpb.br/ojs/index.php/le/article/view/31361/16723

Pacciolla, A. (2016). Psicologia contemporânea e Viktor Frankl: Fundamentos para uma psicoterapia existencial (S. Cobucci, Trad.). Vargem Grande Paulista, SP: Cidade Nova.

Reker, G. T., \& Cousins, J. B. (1979). Factor structure, construct validity and reliability of the Seeking of Noetic Goals (SONG) and Purpose In Life (PIL) Tests. Journal of Clinical Psychology, 35(1), 85-91. doi: 10.1002/1097-4679(197901)35:13.0.CO;2-R

Scheier, M. F., Wrosch, C., Baum, A., Cohen, S., Martire, L. M., Matthews, K. A., Schulz, R., \& Zdaniuk, B. (2006). The Life Engagement Test: Assessing Purpose in Life. Journal of Behavioral Medicine, 29(3), 291-298. doi: 10.1007/s10865-005-9044-1

Schnell, T. (2009). The Sources of Meaning and Meaning in Life Questionnaire (SoMe): Relations to demographics and well-being. The Journal of Positive Psychology, 4(6), 483-499. doi: 10.1080/17439760903271074

Steger, M. F., Frazier, P., Oishi, S., Kaler, M. (2006, January). The meaning in life questionnaire: Assessing the presence of and search for meaning in life. Journal of Counseling Psychology, 53(1), 80-93. doi: 10.1037/0022-0167.53.1.80

Su, T. J., Chen, H. F., Chen, Y. C., Yang, Y. S., \& Hung, Y. T. (2006). Factors Related to Meaning of Life in Taiwanese Women Treated with In Vitro Fertilization. Journal of the Formosan Medical Association, 105(5), 404-413. doi: 10.1016/S0929-6646(09)60137-6

Timmerman, M. E., \& Lorenzo-Seva, U. (2011). Dimensionality assessment of ordered polytomous items with parallel analysis. Psychological Methods, 16(2), 209-220. doi: 10.1037/a0023353

Valladares, L. M., Zavala, M. A., Tarango, I. I., Medina, M. M. G., \& Medina, M. M. G. (2004). El Sentido de vida en Estudiantes de Primer Semestre de la Universidad de la Salle Bajío. Revista del Centro de Investigación, 6(22), 5-13. Recuperado de http://www.redalyc.org/ articulo.oa?id $=34202201$

Xausa, I. A. M. (2011). A psicologia do sentido da vida. Campinas: Vide Editorial.

\section{Sobre os autores}

Raul Bruno Tibaldi Nascimento é psicólogo graduado pela Universidade Federal de Mato Grosso, com ênfase em Intervenções em Processos de Saúde e Sofrimento Psíquico e experiência em atendimentos psicoterapêuticos individuais e em plantão psicológico. Âreas de interesse: Logoterapia e Análise Existencial; Cuidados Paliativos.

Tatiane Lebre Dias é graduada em Psicologia. Docente do Programa de Pós-Graduação em Psicologia e do Programa de Pós-Graduação em Educação da Universidade Federal de Mato Grosso. 